Klemola 30, a compact group of four disk galaxies in the southern skies. Gravitational interactions, and perhaps even a recent merger, have generated spiral arms and tidal tails. Luminous matter, presumably stripped from individual galaxies, pervades the group. Computer simulations of such compact groups suggest that all disks will eventually merge into one single remnant resembling a giant elliptical galaxy. Compare this photograph with Fig. $1 b$ of Barnes's paper on page 124 of this issue. (Courtesy of John A. Graham.)

models left the merger remnants rotating too fast.

Most galaxies occur in aggregates ranging from simple pairs through groups to large clusters and superclusters with thousands of members. Among these aggregates, some compact groups of typically 4-6 galaxies separated by only a few galactic radii have been known for several decades (see figure), but interest in these increased dramatically with the publication of two catalogues ${ }^{8.9}$ totalling over 100 such groups. It was soon realized that the putative dark halos surrounding individual galaxies should lead to large crosssections and rapid merging, given that galaxies cross their groups typically in $10^{*}-10^{y}$ years. Why, then, do we still observe compact groups $15 \times 10^{4}$ years after the Big Bang?

The answer probably lies in the general expansion of the Universe. As Kahn and Woltjer ${ }^{11}$ pointed out long ago for our own Local Group of galaxies, the fact that the Milky Way and the Andromeda Galaxy are now approaching each other at 120 kilometres per second suggests strongly that they and other Local Group members are falling back together for the first time since the initial expansion. Similarly, as Barnes argues in this issue ${ }^{4}$, compact groups of galaxies must condense continually from the reservoir of loose groups, which are 50 times more common, through delayed infall helped, of course, by the fierce dynamical friction experienced when members occasionally collide. His simulation indicates that, if the larger two of the six disks were scaled up to the size of the Milky Way, the first two mergers in his compact group would occur within $5 \times 10^{8}$ years and the whole group would have merged into one giant elliptical after only $4 \times 10^{9}$ years. In this scheme, then, present-day compact groups are transient configurations of galaxies in the process of merging, or just relatively late stages of collapses delayed by the initial expansion.

A quick check of recent observations shows no contradictions with the scheme.
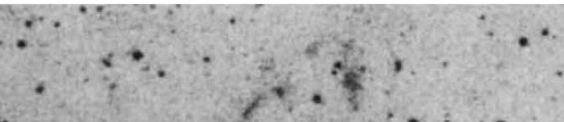

\section{Water everywhere}

LAST week Daedalus invented Wet Paint ${ }^{\circledR}$, a cunning self-cleaning paint. It is mainly a hygroscopic polymer resin, which absorbs water vapour from the night air while the temperature is low and the humidity is high. But in the morning, when the paint warms up again, dispersed waxy globules in the paint melt, expanding strongly as they do so. The strong internal pressure they create in the water-swollen polymer forces water out of it by reverse osmosis. So dirt on the surface is lifted off and washed away daily. In effect, the paint acts as an automatic water-condensing dehumidifier, powered by the temperature difference between day and night.

But Wet Paint is far more than a clever On the contrary, the luminosities of galaxies in Hickson groups ${ }^{11}$ do, indeed, add up to total luminosities typical of giant ellipticals, the predicted end product of group evolution. Also, measured colours of group ellipticals" indicate an unusually high percentage of blue ellipticals, pointing to recent collisions that formed masses of young blue stars. Perhaps most telling is spectroscopic evidence for kinematic perturbations recently found by V.C. Rubin, D.A. Hunter and W.K. Ford (in preparation); eight out of nine brightest galaxies in Hickson' groups show severely perturbed rotation patterns suggestive of mergers, whereas only about one-third of the lesser group members show similar patterns.

Compact groups are thus emerging as the third believable source of delayed galaxy formation and transformation, after the disk-disk mergers reviewed by Toomre $^{12}$ and the many ongoing mergers of gas-rich galaxies revealed by the Infrared Astronomical Satellite (IRAS) about 5 years ago. Mergers, once perceived as rare oddities, now seem to be ubiquitous. Classifying galaxy morphologies 60 years ago, Hubble could scarcely have guessed how fleeting the shapes of galaxies may be. Today, the special thrill provided by Barnes's numerical experiment is to make us bystanders in a cosmic game of Russian roulette; yet instead of death from hits, there is rebirth and growth.

François Schweizer is in the Department of Terrestrial Magnetism, Carnegie Institution of Washington, Washington DC 20015, USA.
Toomre
(1972).
2. Negroponte, J. \& White, S.D. Mon. Not. R. astr. Soc. 205 1009-1029 (1983).
3. Barnes, J.E. Astrophys. J. 331, 699-717 (1988)
4. Barnes, J.E. Nature 338, 123-126 (1989)
5. Edmunds, M. G. Nature 337, 600-601 (1989)
6. Schweizer, F. Science 231, 227-234 (1986).
7. Bender, R. Astr. Astrophys. 202, L5-L8 (1988)
8. Rose, J.A. Astrophys. J. 211, 311-318 (1977).
9. Hickson, P. Astrophys. J. 255, 382-391 (1982).
10. Kahn, F.D. \& Woltjer, L. Astrophys. J. 130. 705-717 (1959).
11. Hickson, P., KindI, E. \& Auman, J.R. Astrophys. J. Suppl. (in the press)
12. Toomre, A. in The Evolution of Galaxies and Stellar Popu- lations (eds Tinsley, B.M. \& Larson, R.B.) 401-416 (Yale University Obsenvatory, New Haven, 1977) way of keeping cars clean and lifting graffiti. Daedalus recalls the many areas of the world, from California to Libya, where water has to be expensively piped from hundreds of miles away, or mined from shrinking and increasingly contaminated ground-water reserves. The atmosphere contains about 1 per cent by weight of water, freely available to anyone with the wit to extract it. Wet Paint now provides that wit.

A square metre of Wet Paint should capture every day about a litre of water. So ten square metres or so could keep a man alive and tolerably clean. A hundred square metres would permit him almost an urban lifestyle. DREADCO's engineers are already devising Wet-Painted water towers whose extensive surfaces accumulate water efficiently from the night breezes, and deliver it automatically, filtered from airborne dust and dirt, every morning. They will need no power and consume no resources. At last every home and rural village can be aqueously self-sufficient.

A small-scale, distributed water source like Wet Paint will never meet the needs of heavy industry. Steel works and chemical plants will still need to be sited near lakes or rivers. But some agriculture could benefit. A desert would have to be covered several times over with Wet Paint to gain the water equivalent of normal temperate rainfall. But a Wet-Painted greenhouse, trapping and recycling the water naturally transpired by the plants within, could well flourish in such a desert. It could help an isolated and parched community to provide its own food as well as its own water.

On a small scale too, Wet Paint water condensers will find many uses. With their aid, cats and office plants and laboratory rats will be regularly supplied with water, batteries and aquaria will be topped up, sandwiches kept moist. Campers and explorers will bless the reliability and purity of their product; and paranoid tourists, warned not to drink the local water, will at last have an alternative to whisky.

David Jones 